\title{
FAK regulates platelet extravasation and tumor growth after antiangiogenic therapy withdrawal
}

\author{
Monika Haemmerle, ${ }^{1}$ Justin Bottsford-Miller, ${ }^{1}$ Sunila Pradeep, ${ }^{1}$ Morgan L. Taylor, ${ }^{1}$ Hyun-Jin Choi, ${ }^{1}$ Jean M. Hansen, ${ }^{1}$ \\ Heather J. Dalton, ${ }^{1}$ Rebecca L. Stone, ${ }^{1}$ Min Soon Cho, ${ }^{2}$ Alpa M. Nick, ${ }^{1}$ Archana S. Nagaraja, ${ }^{1}$ Tony Gutschner, ${ }^{3}$ \\ Kshipra M. Gharpure, ${ }^{1}$ Lingegowda S. Mangala, ${ }^{1}$ Rajesha Rupaimoole, ${ }^{1}$ Hee Dong Han, ${ }^{1}$ Behrouz Zand, ${ }^{1}$ \\ Guillermo N. Armaiz-Pena, ${ }^{1}$ Sherry Y. Wu, ${ }^{1}$ Chad V. Pecot, ${ }^{4}$ Alan R. Burns, ${ }^{5}$ Gabriel Lopez-Berestein, ${ }^{6,7}$ \\ Vahid Afshar-Kharghan, ${ }^{2}$ and Anil K. Sood ${ }^{1,7,8}$ \\ 'Department of Cynecologic Oncology and Reproductive Medicine, ${ }^{2}$ Section of Benign Hematology, and ${ }^{3}$ Department of Cenomic Medicine, The University of Texas MD Anderson Cancer Center, \\ Houston, Texas, USA. “Department of Molecular Therapeutics, University of North Carolina Lineberger Comprehensive Cancer Center, School of Medicine, Chapel Hill, North Carolina, USA. \\ ${ }^{5}$ University of Houston, College of Optometry, Houston, Texas, USA. ${ }^{6}$ Department of Experimental Therapeutics, ${ }^{7}$ Center for RNA Interference and Non-Coding RNA, and ${ }^{8}$ Department of Cancer Biology, \\ The University of Texas MD Anderson Cancer Center, Houston, Texas, USA.
}

Recent studies in patients with ovarian cancer suggest that tumor growth may be accelerated following cessation of antiangiogenesis therapy; however, the underlying mechanisms are not well understood. In this study, we aimed to compare the effects of therapy withdrawal to those of continuous treatment with various antiangiogenic agents. Cessation of therapy with pazopanib, bevacizumab, and the human and murine anti-VECF antibody B20 was associated with substantial tumor growth in mouse models of ovarian cancer. Increased tumor growth was accompanied by tumor hypoxia, increased tumor angiogenesis, and vascular leakage. Moreover, we found hypoxia-induced ADP production and platelet infiltration into tumors after withdrawal of antiangiogenic therapy, and lowering platelet counts markedly inhibited tumor rebound after withdrawal of antiangiogenic therapy. Focal adhesion kinase (FAK) in platelets regulated their migration into the tumor microenvironment, and FAK-deficient platelets completely prevented the rebound tumor growth. Additionally, combined therapy with a FAK inhibitor and the antiangiogenic agents pazopanib and bevacizumab reduced tumor growth and inhibited negative effects following withdrawal of antiangiogenic therapy. In summary, these results suggest that FAK may be a unique target in situations in which antiangiogenic agents are withdrawn, and dual targeting of FAK and VECF could have therapeutic implications for ovarian cancer management.

\section{Introduction}

Antiangiogenic therapies were conceived and developed based on the discovery that tumor growth relies on neovascularization $(1,2)$. These therapies are approved for treatment of a variety of human cancers (3) and include the monoclonal anti-VEGF antibody bevacizumab $(4,5)$ and multitargeted receptor tyrosine kinase inhibitors (6). However, in most clinical trials, these agents have only offered modest improvements in progression-free survival, without affecting overall survival (7). More interestingly, clinical trials with antiangiogenesis drugs and chemotherapy have shown that effects on survival coincide precisely with duration of bevacizumab treatment, and the progression-free survival curves tend to collapse or even crossover following cessation of the antiangiogenesis drug (8-10). Therefore, concerns have been raised about a

Authorship note: M. Haemmerle and J. Bottsford-Miller contributed equally to this work Note regarding evaluation of this manuscript: Manuscripts authored by scientists associated with Duke University, The University of North Carolina at Chapel Hill, Duke-NUS, and the Sanford-Burnham Medical Research Institute are handled not by members of the editorial board but rather by the science editors, who consult with selected external editors and reviewers.

Conflict of interest: The authors have declared that no conflict of interest exists. Submitted: October 14, 2015; Accepted: February 24, 2016.

Reference information: / Clin Invest. 2016;126(5):1885-1896. doi:10.1172/JCI85086. possible rebound in tumor growth after discontinuation of antiangiogenic agents such as bevacizumab, and studies in patients with ovarian cancer are ongoing in which the effects of long-term versus short-term bevacizumab treatment are further evaluated (NCT01462890 and NCT01802749; https://clinicaltrials.gov/). Intriguingly, rapid vascular regrowth $(11,12)$ and an increase of Ki67 and tumor grade (13) were seen in patients after withdrawal of antiangiogenic agents. Additionally, preclinical models suggest that antiangiogenic therapy inhibited primary tumor growth but resulted in increased metastatic potential of tumors through poorly understood mechanisms (14-16).

Our experimental findings implicate a prominent role for platelet extravasation in the rebound tumor growth following cessation of antiangiogenesis therapy. The clinical observation that thrombocytosis is frequently associated with cancer was made more than 100 years ago $(17,18)$. Recent studies indicate that platelets are integral elements of the tumor microenvironment; platelets were shown to be crucial for tumor growth (19), chemoresistance (20), and metastasis $(21,22)$. Additionally, patients with ovarian cancer with an increased number of platelets at the time of initial diagnosis have significantly worse overall and progressionfree survival $(20,23)$. We recently discovered a novel mechanism whereby paraneoplastic thrombocytosis is a result of a paracrine 
A
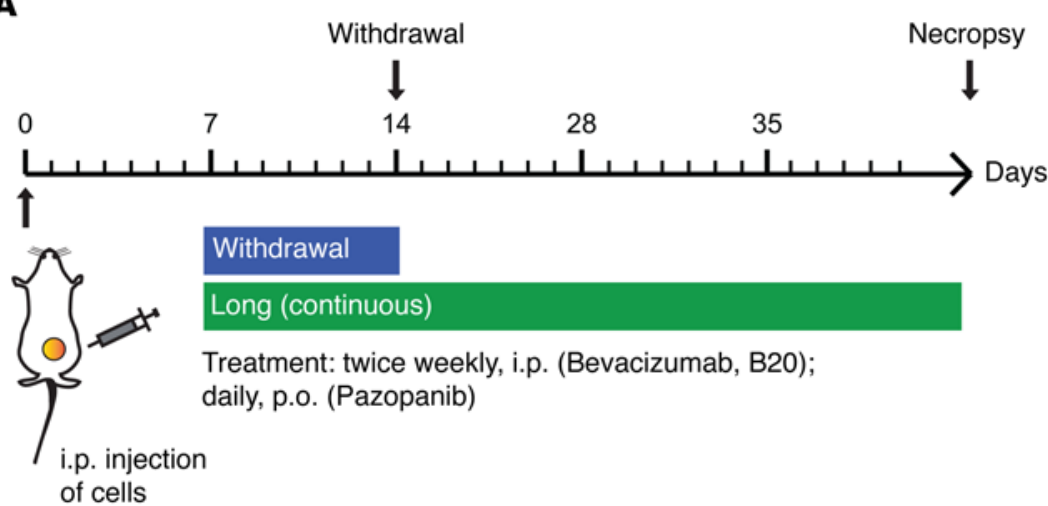

\section{Withdrawal}

\section{Long (continuous)}

Treatment: twice weekly, i.p. (Bevacizumab, B20); daily, p.o. (Pazopanib)

C

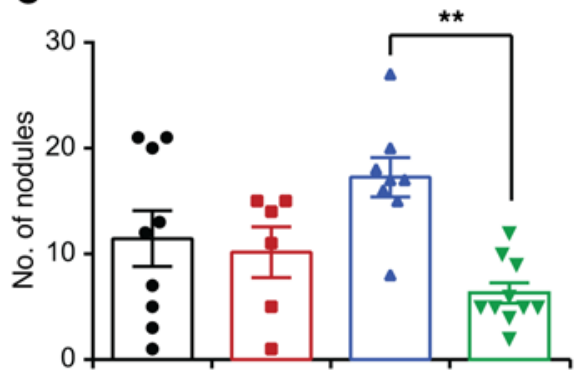

$\square$ Control

Paz pretreatment $\square$ Long Paz

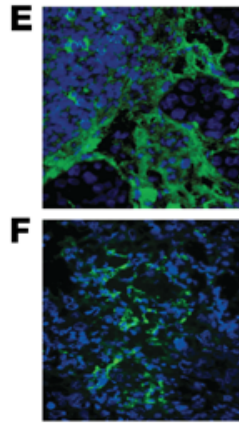

Control

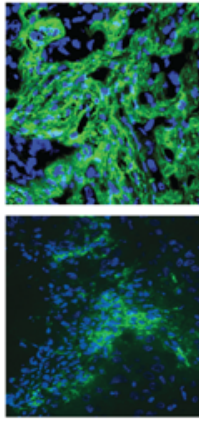

Withdrawal

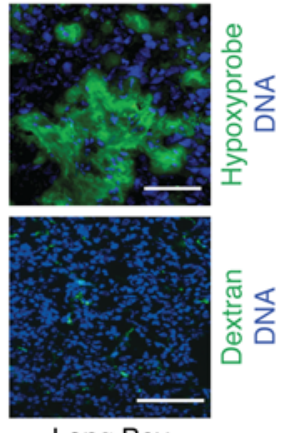

Long Bev

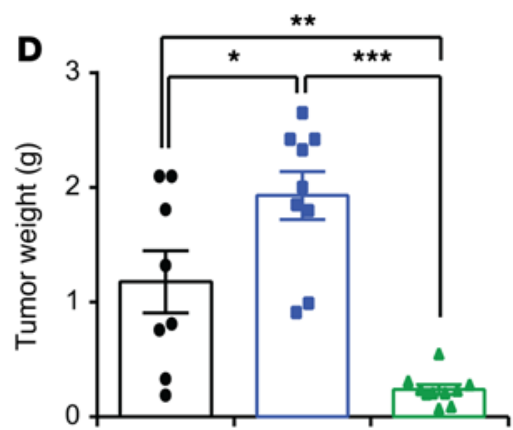

Control Withdrawal Long Bev
B

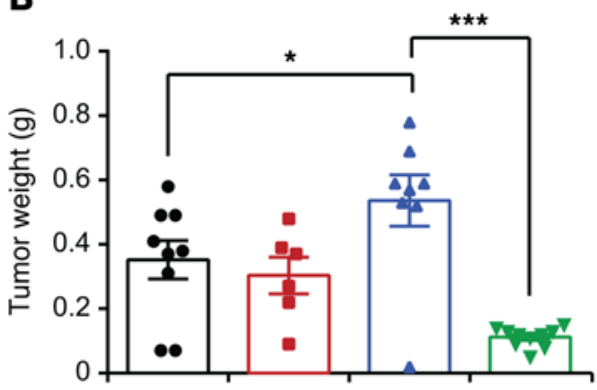

Control

Paz pretreatment $\square$ Long Paz
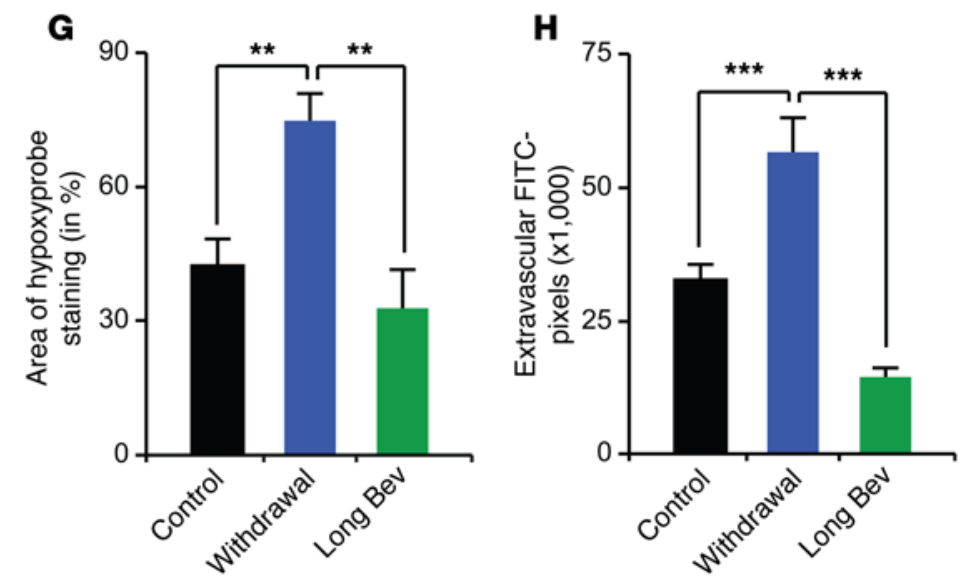
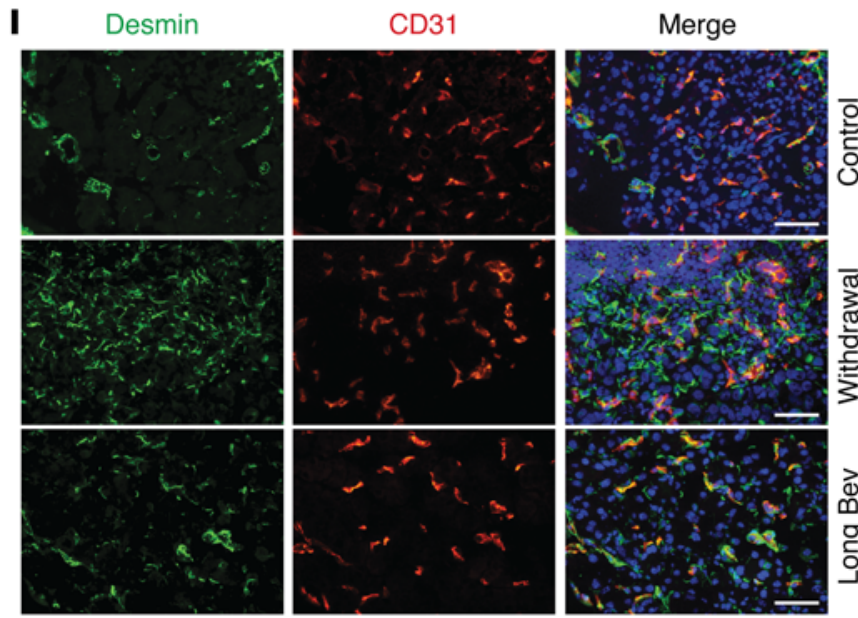

$\mathbf{J}$
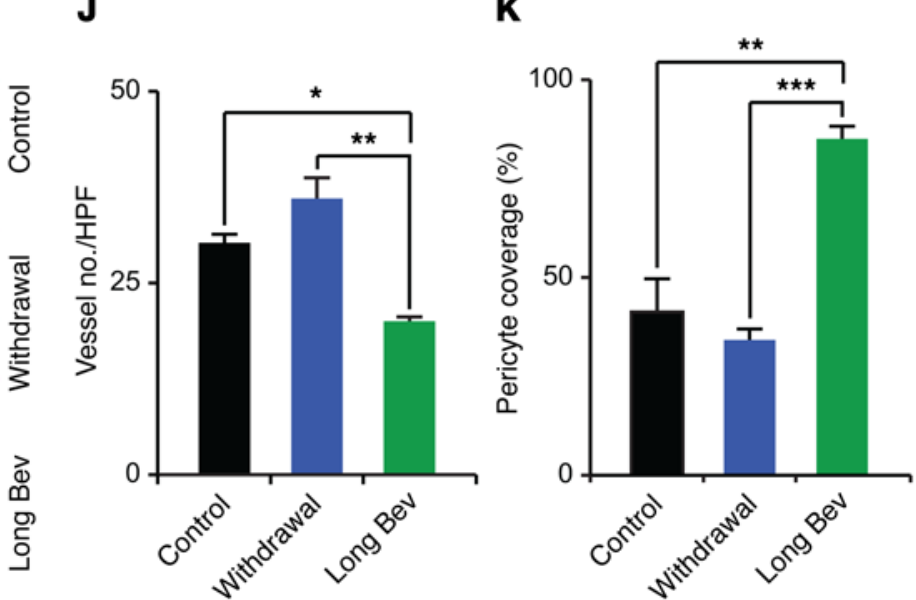
Figure 1. Tumor rebound after cessation of antiangiogenic therapy.

(A) Therapeutic schema for withdrawal and continuous treatment with antiangiogenic therapy. (B) Mean aggregate tumor weight and (C) number of nodules of tumors induced by i.p. injection of SKOV3ip1 human ovarian cancer cells into nude mice with various antiangiogenic treatment schedules. (D) Mean aggregate tumor weight of 2774 ovarian tumors after bevacizumab withdrawal or continuous treatment. (E) Visualization of tumor hypoxia and (F) vascular permeability after i.v. injection of hypoxyprobe and dextran, respectively. Scale bar: $100 \mu \mathrm{m}$. (G and $\mathbf{H})$ The quantification of hypoxyprobe staining and the number of $\mathrm{FITC}^{+}$dextran pixels after indicated treatments. (I-K) Vessel number and pericyte coverage after antiangiogenic therapy withdrawal and continuous treatment. (I) Representative immunofluorescence staining and (J and $\mathbf{K}$ ) bar graphs. HPF, high-powered field. Scale bar: $100 \mu \mathrm{m}$. (B-D) $n=8-10$ mice per group. (E, F, and I) Representative images from at least 5 mice per group. ${ }^{*} P<0.05,{ }^{* *} P<0.01$, ${ }^{* * *} P<0.001$ (1-way ANOVA followed by a Tukey's multiple comparison post-hoc test in $\mathbf{B}-\mathbf{D}, \mathbf{G}, \mathbf{H}, \mathbf{J}$, and $\mathbf{K})$. Averaged data are presented as the mean \pm SEM. Paz, pazopanib; Bev, bevacizumab.

circuit of thrombopoietic cytokines in tumor and host tissue (23). We found that platelets extravasate from the tumor microvasculature into the tumor microenvironment (23), in which they can release cytokines with angiogenic and mitogenic roles and serve as important regulators of angiogenesis $(24,25)$. We believe that our findings demonstrate a pivotal role for platelets in stimulating rebound tumor growth and identify novel therapeutic opportunities to block such effects.

\section{Results}

Withdrawal of antiangiogenic therapy results in accelerated tumor growth. To determine the impact of withdrawal of different antiangiogenic agents compared with continuous treatment in orthotopic mouse models of ovarian cancer, we carried out a set of experiments in which we injected mice i.p. with various human or mouse ovarian cancer cells (day 0). These mice were randomized into three groups: control; withdrawal of antiangiogenic therapy (treatment from day 7-14); and continuous antiangiogenic therapy (treatment from day 7 until necropsy) (Figure 1A). Withdrawal of treatment with pazopanib (a multitargeted receptor tyrosine kinase inhibitor) in mice bearing SKOV3ip1 tumors resulted in a significant increase in tumor growth as compared with that in the control group, whereas continuous pazopanib treatment significantly reduced the aggregate tumor weight as well as the number of tumor nodules (Figure 1, B and C). We observed similar results using different ovarian cancer models and different antiangiogenic agents: pazopanib in HeyA8 tumors (Supplemental Figure 1, A and B; supplemental material available online with this article; doi:10.1172/JCI85086DS1) and bevacizumab (a humanized monoclonal anti-VEGF antibody) in the 2774 tumors (Figure 1D and Supplemental Figure 1, C and D). After withdrawal of antiangiogenic therapy, i.p. tumor nodules induced by the 2774 ovarian cancer cells exhibited increased areas of hypoxia, as detected by staining for pimonidazole adduct formation (Figure 1, E and G), and increased vascular leakage, as determined by extravascular FITC-dextran (Figure 1, F and H). Moreover, analysis of $\mathrm{CD} 31^{+}$microvessels (Figure 1, I and J) and desmin $^{+}$pericytes covering endothelial cells (Figure 1, I and K) showed increased microvessel density but reduced pericyte coverage after withdrawal of the antiangiogenic drug.
Increased platelet infiltration into tumors after withdrawal of antiangiogenic therapy. We and others have shown that platelets enhance tumor growth $(19,23,26)$, chemoresistance (20), and metastasis $(21,22)$. Although platelet number and function can be altered by hypoxia (27-29), the functional effects of hypoxia in the tumor microenvironment on platelets are unknown. Therefore, we examined whether platelet infiltration into the tumor microenvironment could contribute to tumor growth following cessation of antiangiogenic therapy. Withdrawal of bevacizumab treatment led to a 3.5-fold increase in platelet infiltration compared with that after control or long-term bevacizumab treatment (Figure 2, A and B). Platelets attached to endothelial cells and extravasated in between endothelial cells into the tumor microenvironment (Supplemental Figure 2, A and B). Given the role of ADP as a potent platelet agonist $(30,31)$ and the hypoxia resulting from antiangiogenic drugs, we investigated the effect of hypoxia on ADP secretion by cancer cells. SKOV3ip1 ovarian cancer cells grown in a hypoxic environment (1\% oxygen) for 24, 48, and 72 hours produced 2 -fold, 4.3 -fold, and 5.6-fold more ADP than cells grown under normoxia (21\% oxygen), respectively, as was determined by measuring ADP concentration in serum-free cell culture media incubated with cancer cells (Figure $2 \mathrm{C}$ ). Likewise, in vivo tumors resected from mice exposed to bevacizumab for 7 days had significantly higher ADP concentrations than either the untreated controls or tumors continuously treated with bevacizumab (Figure 2D). To further assess how platelets regulate tumor cell characteristics, various ovarian cancer cells were exposed to platelets. Coincubation significantly increased tumor cell proliferation (Figure 2E), which was dependent on platelet activation (Figure 2F). Activation of platelets by tumor cells was demonstrated by surface expression of P-selectin (also known as CD62P) and the active form of CD41/CD61 (also known as GpIIb/ IIIa complex). These results showed that $23 \%( \pm 8.5 \%)$ of platelets were positive for P-selectin and $37 \%( \pm 15.5 \%)$ were positive for the GpIIb/IIIa complex after coincubation with tumor cells (Supplemental Figure 2C). These findings are supported by a prior report (32). Additionally, platelets also significantly decreased apoptosis (Supplemental Figure 2D) and increased tumor cell invasion (Supplemental Figure 2E).

Platelets are decisive for tumor outgrowth after withdrawal of antiangiogenic therapy. Next, we examined the effect of platelet counts on tumor growth and the response to antiangiogenic therapy. To alter platelet counts, we induced thrombocytosis by transfusion of platelets and thrombocytopenia by i.v. injection of the monoclonal anti-glycoprotein $\mathrm{Ib} \alpha(\mathrm{GPIb} \alpha)$ antibody twice weekly, respectively. Mice carrying A2780ip2 tumors were randomized to the following groups: untreated control, platelet transfused, and platelet depleted by antiplatelet antibody (APA). Platelet counts were measured at the time of necropsy (Supplemental Figure 3A). Mice receiving platelet transfusions twice weekly had on average 1.9- and 5.4-fold higher tumor weight compared with the control group and with mice treated with APA twice weekly, respectively (Figure 3A). Similar results were obtained in the SKOV3ip1 model (Supplemental Figure 3B). Tumors resected from platelet-transfused mice showed significantly higher proliferation (Figure 3B) and a lower apoptosis rate (Figure 3C) compared with those of control mice, as measured by the number of $\mathrm{Ki} 67^{+}$tumor cell nuclei and the number of cleaved caspase-3-positive tumor cells, respectively. 


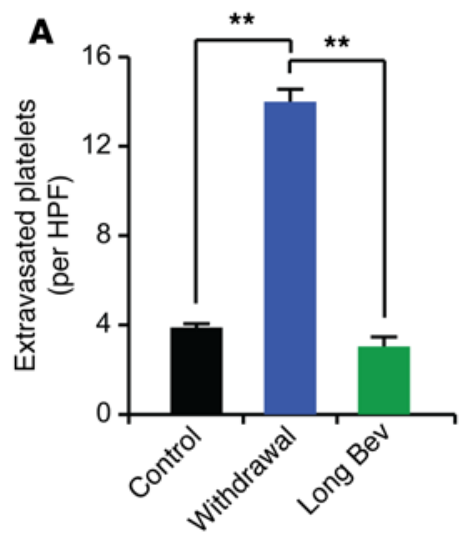

B
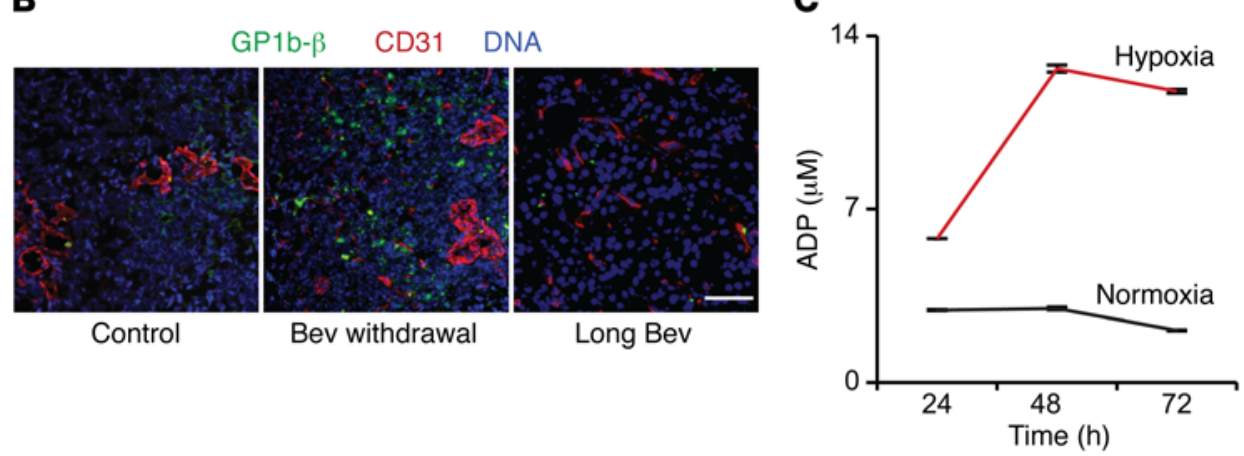

E
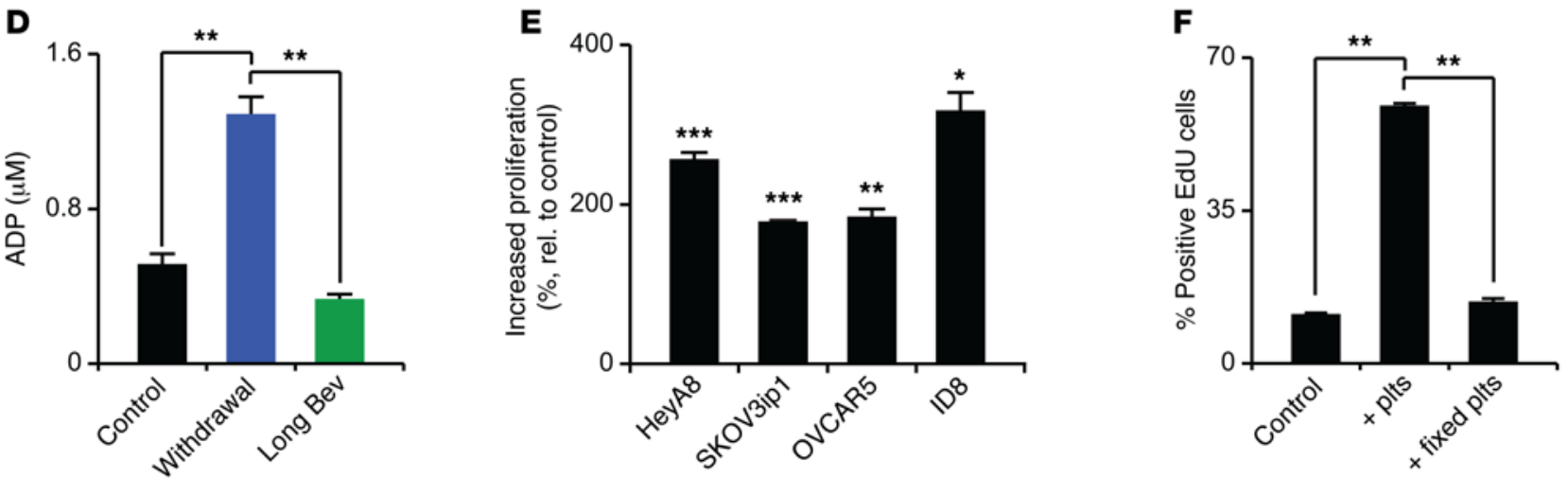

Figure 2. Antiangiogenic treatment, hypoxia, and platelet effect on cancer cells. (A) The number of extravasated platelets in 2774 ovarian cancer cellinduced tumors in control mice and mice exposed to bevacizumab withdrawal or long-term treatment. (B) Representative immunofluorescence staining of tumors resected from control mice and mice exposed to bevacizumab withdrawal and long-term bevacizumab treatment. CD31 (red) is an endothelial marker, and GPIb $\beta$ (green) is a platelet marker. Scale bar: $100 \mu \mathrm{m}$. (C) ADP concentrations ( $\mu \mathrm{M}$ ) in supernatants of SKOV3ip1 cells grown in hypoxia (1\% oxygen) or normoxia ( $21 \%$ oxygen) for 24,48 , and 72 hours $(n=3)$. (D) ADP levels $(\mu M)$ in SKOV3ip1 tumors resected from mice exposed to long-term bevacizumab or withdrawal of bevacizumab. (E) Increase in proliferation of different ovarian cancer cell lines after exposure to platelets for 72 hours, as measured by EdU incorporation $(n=3)$. (F) Proliferation of HeyA8 ovarian cancer cells after coincubation with normal or $1 \%$ paraformaldehyde-fixed platelets $(n=3)$. (A and B) Quantification and representative images from tumors of at least 5 mice per group. (D) Quantification of ADP levels in tumors of 2 mice per group. ${ }^{*} P<0.05$, ${ }^{* *} P<0.01$, ${ }^{* *} P<0.001$ (1-way ANOVA followed by a Tukey's multiple comparison post-hoc test in A, D, and F; 2-tailed Student's $t$ test in $\mathbf{E})$. Averaged data are presented as the mean \pm SEM.

To determine whether transfused platelets were infiltrating into tumors, mice with SKOV3ip1 tumors were injected with $300 \times 10^{6}$ platelets from Pf4-Cre-yellow fluorescence protein (Pf4-Cre-YFP) mice, which allowed for specific labeling of the megakaryocytic lineage, including platelets with YFP (23). Representative immunofluorescence images showed that i.v.-infused platelets were present in tumor tissues (Supplemental Figure 3C). Moreover, direct and indirect coculture of HeyA8 ovarian cancer cells with platelets showed that HeyA8 cells directly interacting with either intact or lysed platelets substantially increased their proliferation rate. However, the proliferation rate of cancer cells that were separated from platelets by a dense endothelial monolayer was almost equal to that of control cells that were not exposed to platelets (Supplemental Figure 3D). To investigate the relationship between platelet counts and accelerated tumor growth after withdrawal of antiangiogenic therapy, tumor-bearing nude mice were subjected to withdrawal of antiangiogenic therapy in combination with APA for platelet depletion. Lowering platelet counts completely abolished rebound in tumor growth after antiangiogenic withdrawal in both A2780ip2 and HeyA8 tumor models and maintained the tumor weight at the same level as that in mice receiving continuous antiangiogenic therapy (Figure 3, D and E). To further investigate our hypothesis that platelets are crucial for the rebound of tumor growth after withdrawal of antiangiogenic therapy, we performed an additional in vivo experiment. Mice were injected with A2780ip2 ovarian cancer cells and randomized into three groups: control IgG; APA alone; and APA plus bevacizumab withdrawal. APA treatment was started on the day of tumor cell injection and was continued until the end of the experiment. In the withdrawal group, treatment with bevacizumab was started 1 week after tumor cell injection and withdrawn after a week of treatment. Supplemental Figure 3E shows that tumor weight was reduced by $82 \%$ in the group that received APA alone and by $67 \%$ in the group that received APA plus bevacizumab withdrawal. Although tumor weight was slightly higher in the group that received APA plus bevacizumab withdrawal, there was no significant difference compared with the group that received APA alone. These results highlight platelets as important mediators of tumor progression after cessation of antiangiogenic therapy. Platelets are known to store and secrete various tumorigenic and angiogenic proteins (24) that can promote tumor growth. To determine whether the quantity of angiogenic proteins changes in the withdrawal setting, we treated mice with A2780ip2 i.p. tumors with bevacizumab 


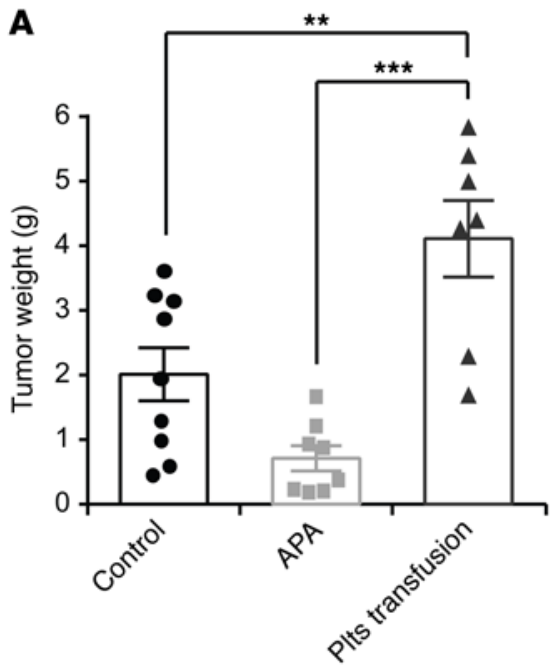

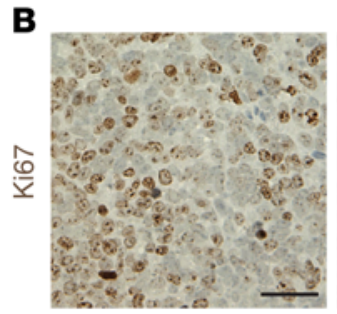

Control

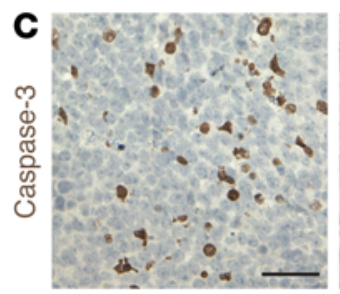

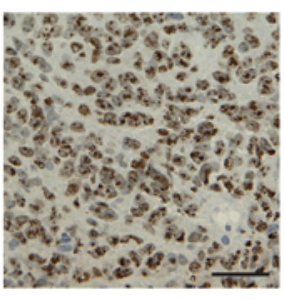

Transfusion

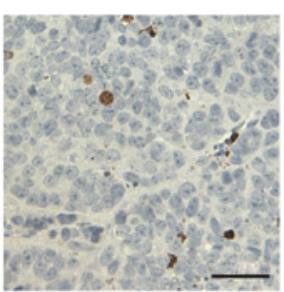

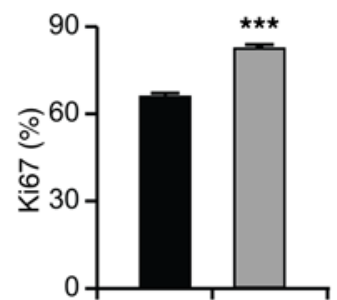

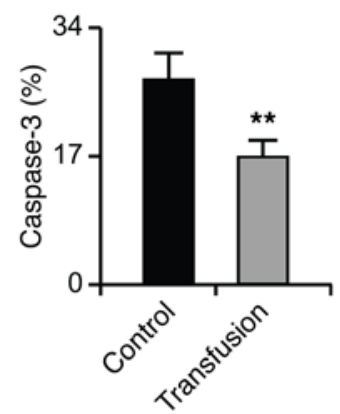

D

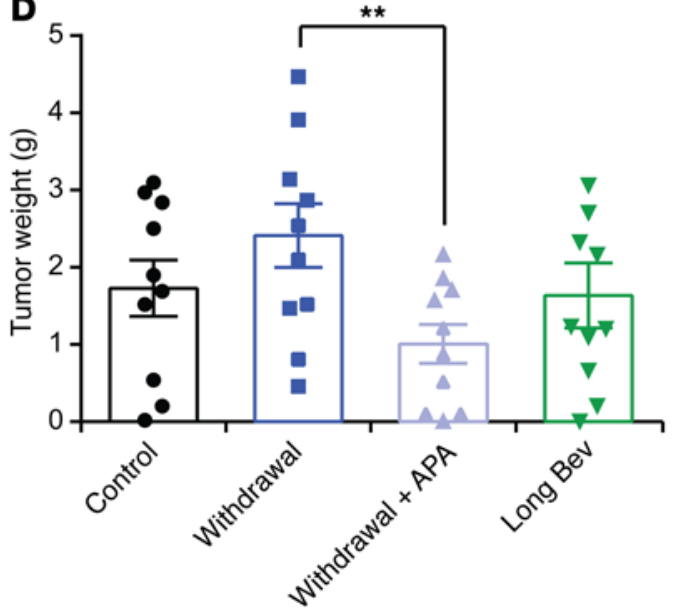

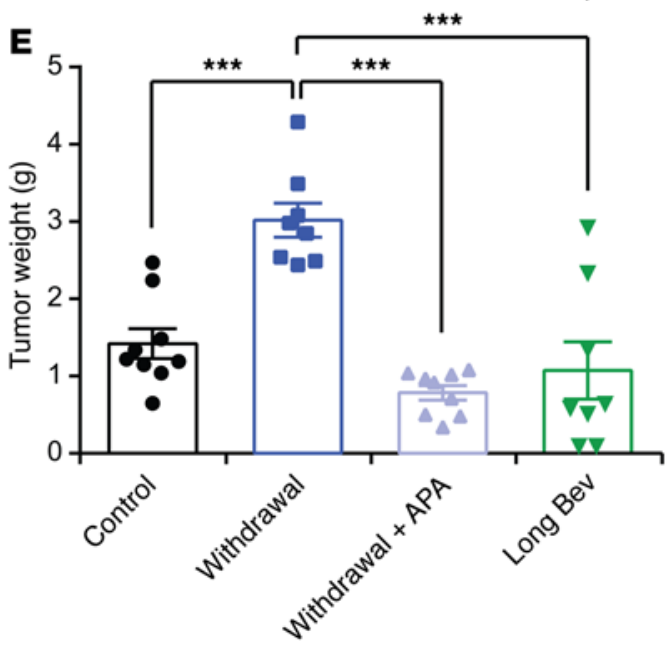

Figure 3. Platelets are important mediators of tumor rebound growth after withdrawal of antiangiogenic therapy. (A) Mean aggregate tumor weight of A2780 cell-induced tumors in mice receiving platelet transfusions or APA twice weekly. (B and C) Representative immunohistochemical staining for (B) Ki67 and (C) caspase-3 and their quantification in control tumors (black bars) and tumors transfused with platelets (gray bars) twice weekly. Scale bar: $50 \mu \mathrm{m}$. (D and E) Mean aggregate tumor weight in (D) A2780 cell- or (E) HeyA8 cell-induced i.p. tumors for each indicated treatment group. (A, D, and E) $n=8-10$ mice per group. (B and C) Representative images from tumors of at least 5 mice per group. ${ }^{* *} P<0.01,{ }^{* * *} P<0.001$ (1-way ANOVA followed by a Tukey's multiple comparison post-hoc test in $\mathbf{A}$, D, and $\mathbf{E} ; 2$-tailed Student's $t$ test in $\mathbf{B}$ and $\mathbf{C}$ ). Averaged data are presented as the mean \pm SEM.

for the length of the entire experiment or for 7 days with subsequent withdrawal. Platelet proteins were isolated from each group at the end of the experiment. Evaluation of 53 angiogenesis-related proteins in platelets revealed that the quantity of the analyzed proteins was not significantly different between the two groups (Supplemental Figure 3F). Therefore, it is likely to be the number of platelets infiltrating into the tumor microenvironment and directly interacting with cancer cells, and not the quantity of angiogenic proteins, that is the crucial factor for tumor rebound growth after withdrawal of antiangiogenic drugs.

Platelet expression of focal adhesion kinase is crucial for platelet migration. An important regulator of shape change and movement of platelets is focal adhesion kinase (FAK, also known as protein tyrosine kinase 2 [PTK2]) (33). FAK is overexpressed in a variety of human cancers (34) and is at the interface of tumor cells and their microenvironment (35). To investigate the effect of FAK in the murine host on the rebound growth of the inoculated tumors after antiangiogenic withdrawal, we inhibited FAK expression in nude mice carrying HeyA8 human ovarian cancer cell-induced tumors using a mouse-specific anti-FAK siRNA incorporated in chitosan nanoparticles. Mouse anti-FAK siRNA chitosan nanoparticles reduced expression of FAK in the murine host (including host megakaryocytes and platelets) but did not affect FAK levels in human ovarian cancer cells (Supplemental Figure 4A). Moreover, proliferation and apoptosis of HeyA8 cells was not changed upon transfection with the mouse-specific FAK siRNA (Supplemental Figure 4, B and C). Reducing FAK expression in the host decreased tumor weight by 69\% (Supplemental Figure 4D) and the number of tumor nodules by $56 \%$ (Supplemental Figure 4E) and abrogated tumor growth rebound after withdrawal of antiangiogenic therapy. To further study the effect of platelet FAK, we used platelet-specific FAK-deficient mice 
A
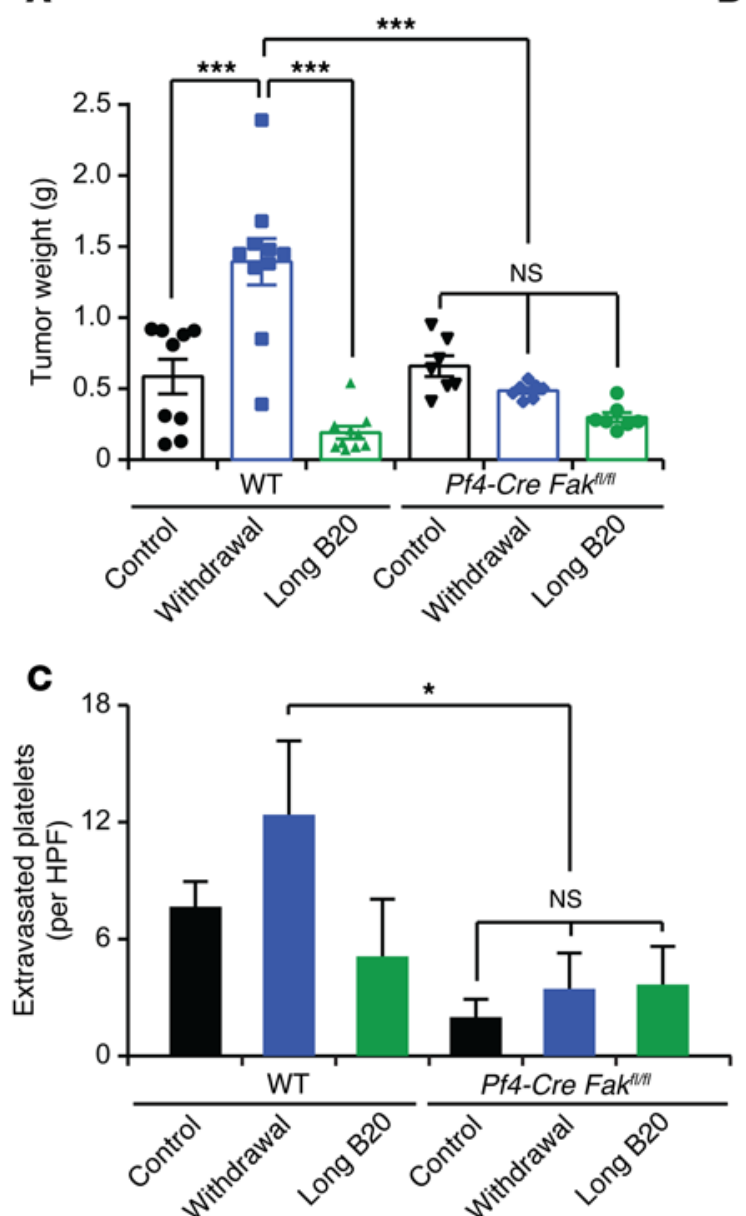

B
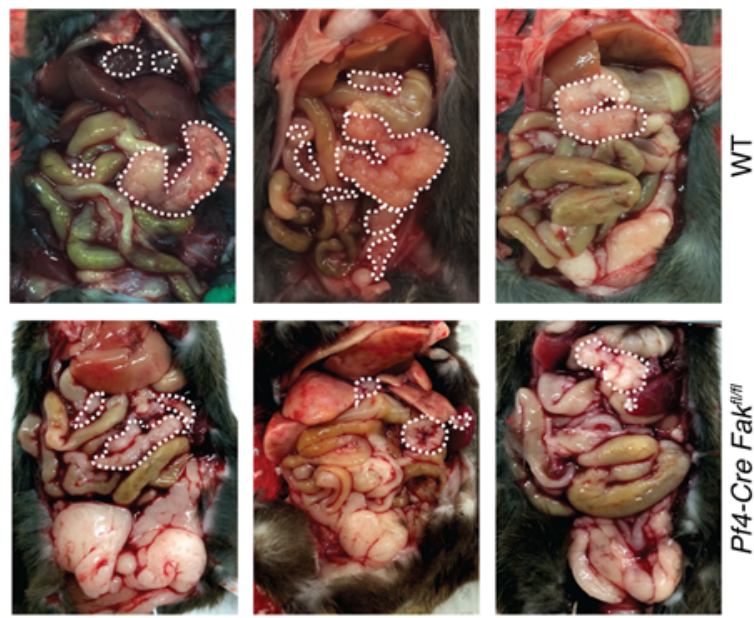

Control

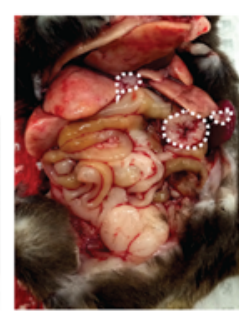

Withdrawal

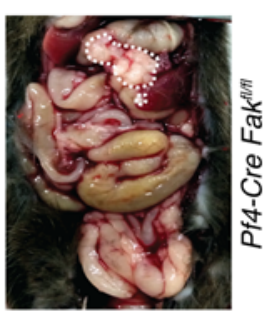

Long B2O

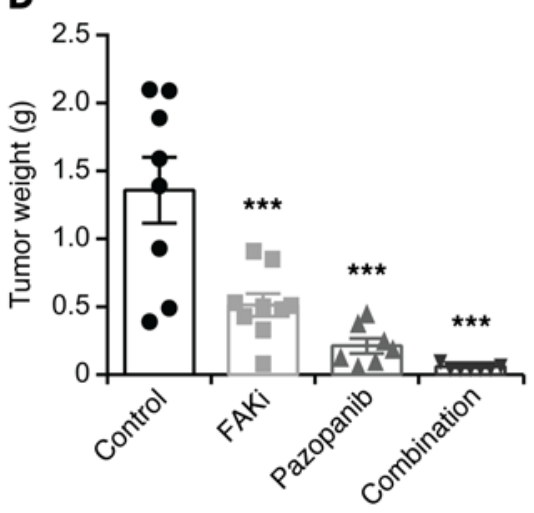

E

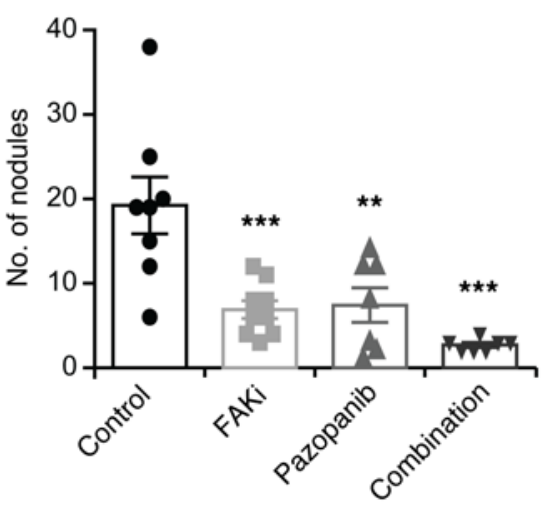

$\mathbf{F}$

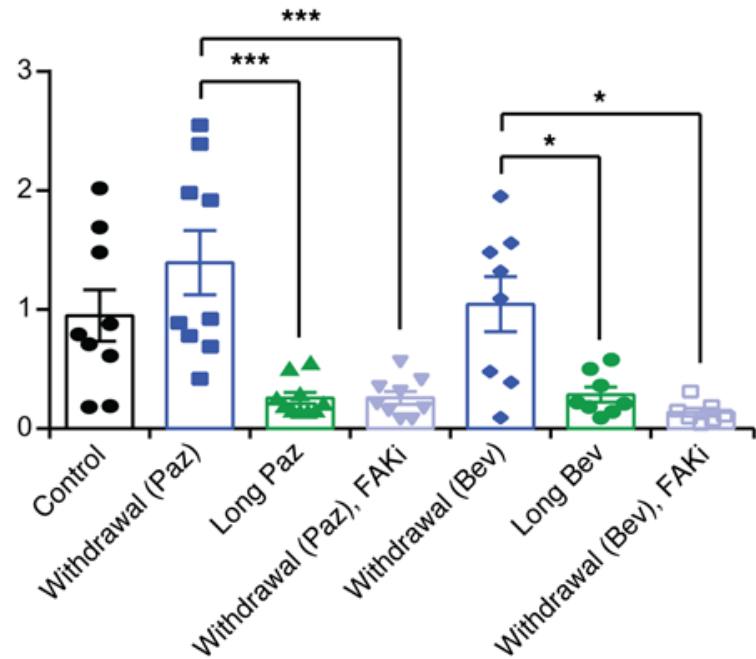

Figure 4. Role of platelet FAK on tumor growth. (A) Mean aggregate tumor weight and (B) representative images of necropsy in WT or platelet-specific FAK-deficient mice that carried tumors induced by i.p. injection of ID8-VEGF murine ovarian cancer cells and were exposed to long-term treatment or withdrawal of anti-VEGF antibody (B20). (C) Quantification of extravasated platelets in ID8-VEGF tumors in WT or platelet-specific FAK-deficient mice. (D) Mean aggregate tumor weight and (E) number of tumor nodules of orthotopic SKOV3ip1 tumors after treatment with pazopanib, the FAK inhibitor GSK2256098 (FAKi), or a combination of the two. (F) Mean aggregate weight of orthotopic SKOV3ip1 tumors after withdrawal or long-term antiangiogenic therapy alone or in combination with FAK inhibitor CSK2256098. (A and D-F) $n=8-10$ mice per group. (B and C) Quantification and representative images of tumors from at least 5 mice per group. ${ }^{*} P<0.05,{ }^{* *} P<0.01,{ }^{* *} P<0.001$ (1-way ANOVA followed by a Tukey's multiple comparison post-hoc test in $\mathbf{A}$ and $\mathbf{C}-\mathbf{F})$. Averaged data are presented as the mean \pm SEM. 

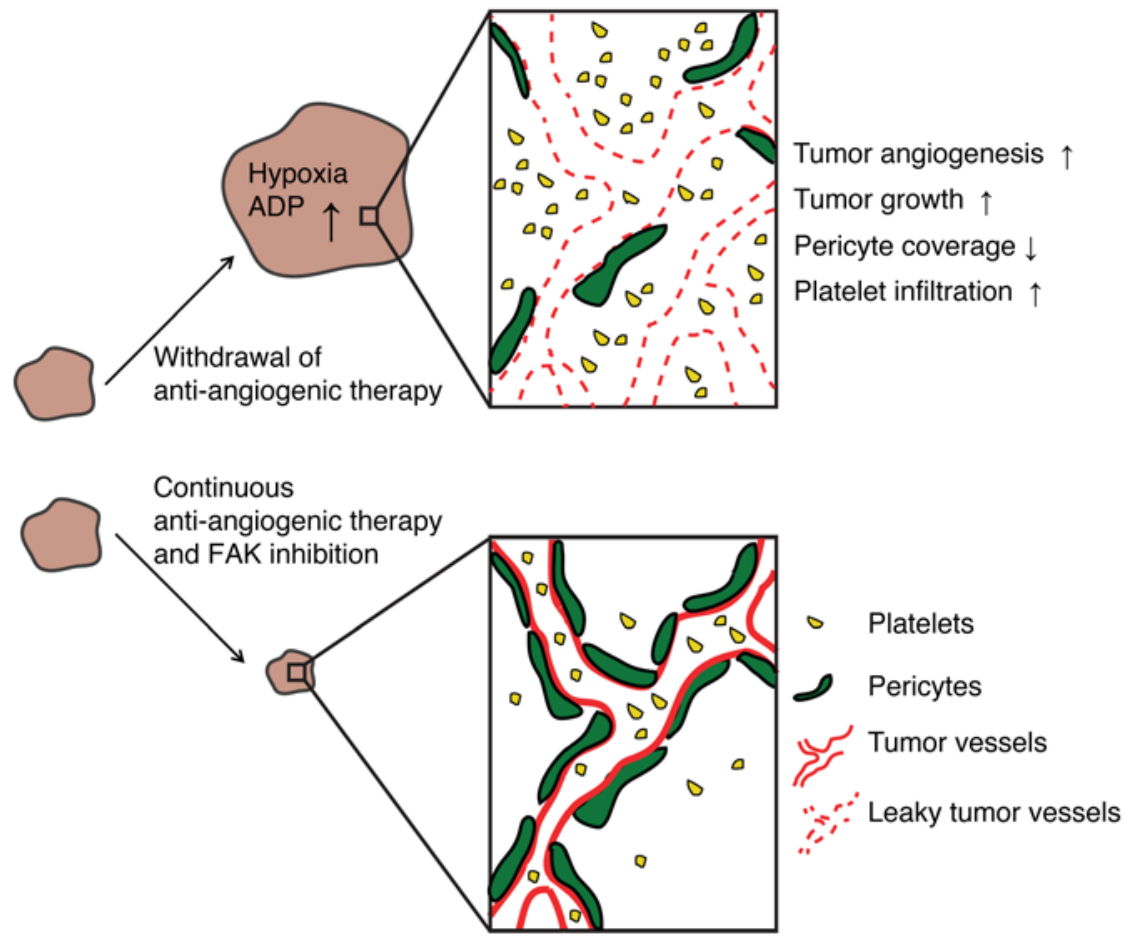

Figure 5. Proposed model of the interactions among antiangiogenic therapy, platelets, and FAK and their effect on tumor growth. Withdrawal of antiangiogenic therapy leads to tumor hypoxia, ADP production, increased angiogenesis, and platelet infiltration, thereby increasing tumor growth. On the other hand, continuous antiangiogenic therapy and/or FAK inhibitors might be a potential regimen to prevent tumor rebound after cessation of antiangiogenic therapy.
(Pf4-Cre Fak ${ }^{f l / f l}$ mice; ref. 33) in our antiangiogenic therapy withdrawal model. We confirmed the absence of FAK protein in platelets isolated from these mice. Furthermore, we isolated $\mathrm{CD} 19^{+} \mathrm{B}$ cells, $\mathrm{CD}^{+} \mathrm{T}$ cells, and $\mathrm{F} 4 / 80^{+}$macrophages from the spleens of Pf4-Cre Fak ${ }^{f l / f l}$ mice using a cell sorter and extracted their RNA. qRT-PCR analysis did not show any significant change in the expression of Fak mRNA in white blood cells of Pf4-Cre Fak ${ }^{f / f l}$ mice (Supplemental Figure 5A). Intriguingly, platelet-specific FAK deficiency prevented a rebound in tumor growth after withdrawal of the anti-VEGF antibody (B20), as was demonstrated by a significant reduction in tumor weight (Figure 4A) and number of tumor nodules (Supplemental Figure 5B). Figure 4B shows representative images of WT and platelet-specific FAK-deficient mice at the time of necropsy. In addition, immunofluorescence staining of resected tumors showed a reduction in platelet infiltration into the tumor microenvironment in platelet-specific FAK-deficient mice as compared with control mice (Figure $4 \mathrm{C}$ and Supplemental Figure 5C). Most notably, we did not detect any increase in platelet infiltration after withdrawal of antiangiogenic therapy in mice with platelet-specific FAK deficiency. Loss of FAK in platelets did not affect the platelet blood counts in the peripheral blood of these mice (Supplemental Figure 5D). Furthermore, ID8-VEGF murine ovarian cancer cells cocultured with WT platelets showed slightly increased proliferation compared with cells exposed to $\mathrm{Fak}^{-1-}$ platelets (Supplemental Figure 5E); however, these effects were minimal. Rates of apoptosis were not different between WT and $\mathrm{Fak}^{-/-}$platelets (Supplemental Figure 5F). These results highlight the fact that the diminished platelet infiltration upon loss of FAK is likely to be the main factor responsible for the rebound in tumor growth after withdrawal of the antiangiogenic therapy.

FAK inhibitors can block tumor rebound after cessation of antiangiogenic therapy. To further investigate the role of FAK on tumor response to antiangiogenic agents, we used a small-molecule inhibitor that targets FAK phosphorylation in orthotopic mouse models of ovarian and uterine cancer $(34,35)$. GSK2256098 is a reversible, highly selective small-molecule inhibitor that targets FAK activity and Y397 phosphorylation $(36,37)$. We assessed the effectiveness of GSK2256098 by measuring FAK ${ }^{Y 397}$ phosphorylation in SKOV3ip1 and HeyA8 cells in vitro (Supplemental Figure 6, A and B). To determine the in vivo effects of GSK2256098, we treated mice carrying SKOV3ip1 tumors with pazopanib, GSK2256098, or a combination of both. Combination of pazopanib and GSK2256098 resulted in a significant reduction in tumor weight (Figure 4D) and the number of tumor nodules (Figure $4 \mathrm{E}$ ) as compared with those in the single-treatment groups. Moreover, FAK inhibition reduced platelet infiltration into the tumor (Supplemental Figure 6C). Similar results were obtained with HeyA8 ovarian cancer cells (Supplemental Figure 6, D and E), although the effect was less pronounced than that in the SKOV3ip1 tumors, probably because of the smaller effect of GSK2256098 on $F A K^{Y 397}$ phosphorylation in these cells. Considering that inhibition of FAK had substantial antitumor effects in combination with an antiangiogenic agent, we investigated whether inhibition of FAK abrogates the rebound in tumor growth after withdrawal of antiangiogenic therapy. Therefore, we performed an experiment in which we administered GSK2256098 after withdrawal of bevacizumab. We found that GSK2256098 treatment of mice completely blocked acceleration in tumor growth after withdrawal of pazopanib or bevacizumab in both SKOV3ip1- (Figure $4 \mathrm{~F}$ and Supplemental Figure 7A) and HeyA8-induced tumors (Supplemental Figure 7, B and C).

\section{Discussion}

In the current study, we have modeled tumor rebound after withdrawal of a variety of antiangiogenic agents. Tumor outgrowth after cessation of treatment was associated with increased intratumoral hypoxia, tumor angiogenesis, vascular permeability, and platelet 
infiltration (Figure 5) as compared with continuous antiangiogenic therapy. Platelet infiltration was dependent on FAK expression, and inhibition of FAK in the platelets itself or by a FAK inhibitor prevented tumor outgrowth after cessation of therapy.

Antiangiogenic agents are used for various human cancers (3). Recently, the human monoclonal anti-VEGF antibody bevacizumab was approved by the FDA for platinum-resistant recurrent ovarian cancer based on a phase III clinical study comparing bevacizumab plus chemotherapy to chemotherapy alone (38-40). However, the effect on progression-free survival was modest. Moreover, studies completed in patients with ovarian cancer in which bevacizumab was given in combination with chemotherapy over either a short time or until disease progression show that shortly after bevacizumab withdrawal survival curves collapse (8-10). These findings are quite distinct from those of prior phase III clinical trials with chemotherapy alone in which progressionfree survival curves consistently remain separate $(41,42)$. These results suggested that, especially in high-risk patients, it might be beneficial to further extend the treatment duration of bevacizumab beyond chemotherapy and even after disease progression. Therefore, ongoing trials (NCT01462890 and NCT01802749; https://clinicaltrials.gov/) are addressing these important questions in ovarian cancer, whereas studies in colorectal and breast cancer already provided evidence of benefit $(43,44)$.

Preclinical studies have shown that antiangiogenic agents induce pronounced hypoxia and in turn might facilitate metastasis (45). However, whether tumor regrowth occurs after discontinuation of antiangiogenic therapy is controversial. Results from a study using various different cell lines indicate that tumors that relapsed after cessation of therapy did not grow faster compared with untreated tumors (46). However, most of this work was done by injecting cancer cells subcutaneously where tumor-related angiogenesis is highly different from that at orthotopic sites (47). Hypoxic tumors have abnormal and highly heterogeneous blood vessels, with disorganized endothelial cells and a lack of pericytes $(48,49)$. Concordant with these findings, we found increased tumor angiogenesis associated with intratumoral hypoxia and vascular leakage after withdrawal of antiangiogenic therapy. This might be counterintuitive, as one would expect that more vessels would theoretically lead to reduced hypoxia. However, our results are supported by those of earlier studies, in which short-term treatment with an anti-VEGFR2 antibody indeed increased hypoxia and HIF-1 $\alpha$ expression in the tumor, which in turn increased tumor angiogenesis (50-52). Increased vascular permeability can lead to platelet infiltration to a great extent (23). Once in the tumor, platelets can secrete a wide variety of prosurvival and proangiogenic cytokines (24). Therefore, it is not surprising that they are crucial for tumor cell survival. Indeed, nearly $40 \%$ of people with platelet counts over 400,000 per cubic millimeter without any evidence of iron deficiency or inflammatory conditions have an occult cancer, most commonly ovarian cancer, among others (53). Aside from the clinic, experimental evidence suggests that platelets can drive tumor progression and metastasis via various mechanisms (54). Our own experiments indicated that platelets transfused into mice indeed homed to the tumor tissue and that direct contact of tumor cells with platelets is crucial for tumor cell proliferation. Furthermore, these results sug- gested that the number of platelets rather than their quantitative protein content is important for tumor rebound after withdrawal of antiangiogenic therapy. Altogether, this implies that reducing platelet levels in the blood can be a potent antiproliferative and antimetastatic strategy $(19,21)$. Indeed, our results indicated that, regardless of the time of administration of APA, reducing platelet counts significantly inhibited tumor rebound after withdrawal of bevacizumab. However, lowering platelet numbers in patients with cancer requires careful titration, as thrombocytopenia can easily lead to life-threatening bleeding complications. Although platelet function and numbers can change under hypoxic conditions $(27-29,55)$, their role in tumor hypoxia, especially with regard to antiangiogenic therapy-induced hypoxia, is not well understood. We showed that increased platelet infiltration after withdrawal of antiangiogenic therapy was dependent on expression of FAK in platelets. Intriguingly, platelet-specific knockout of FAK completely blocked tumor outgrowth after cessation of antiangiogenic therapy. Although tumor weight was not significantly different between the two control groups, platelet infiltration was significantly reduced in tumors of $\mathrm{Pf} 4-\mathrm{Cre}^{+} \mathrm{Fak}^{\mathrm{fl} / \mathrm{fl}}$ mice. One explanation could be that platelets extravasated even before a detectable increase in tumor growth. This is supported in a recent publication by Qi et al. (56), who showed that, in human colorectal adenomas, platelet infiltration can be detected even before the presence of adenocarcinoma; however, the number of infiltrating platelets increased further during carcinoma development. Another possible explanation as to why tumor weights were not significantly different is that the time point of necropsy was dependent on the group of WT mice in which antiangiogenic therapy was withdrawn; these mice became moribund much earlier than the $\mathrm{Pf} 4-\mathrm{Cre}^{+} \mathrm{Fak}^{f l / f l}$ mice. The Pf4-Cre $\mathrm{Fak}^{f l / f l}$ system was published and characterized earlier (33). The authors evaluated the expression of FAK in megakaryocytes and platelets as well as in several other organs. FAK was absent only in megakaryocytes and platelets. However, recent work by Pertuy et al. (57) showed that the Pf4-Cre transgene might have a broader expression beyond the megakaryocytic lineage. Pertuy et al. found that $0.3 \%$ of $\mathrm{CD} 45^{+}$leukocytes as well as a small percentage of intestinal epithelial cells showed expression of Pf4. Despite these results, the authors confirmed that the Pf4-Cre system is extremely valuable and a powerful instrument to study the function of megakaryocytes and platelets in diverse physiological and pathological settings, as also shown by others (58). We confirmed the absence of FAK protein in platelets of Pf4-Cre+ $\mathrm{Fak}^{\mathrm{fl} / \mathrm{fl}}$ mice. Moreover, Fak mRNA expression was not significantly different in various immune cells isolated from the spleens of WT and Pf4$\mathrm{Cre}^{+} \mathrm{Fak}^{f l / f l}$ mice.

Comparable results with regard to tumor growth and tumor rebound after withdrawal of antiangiogenic therapy were found using the FAK inhibitor GSK2256098, which combined well when given together with the antiangiogenic agents pazopanib or bevacizumab. GSK2256098 is a highly specific small-molecule inhibitor for $F A K$, both for humans and mice, and has been designed to block $F A K^{\mathrm{Y} 397}$ phosphorylation which is necessary for kinase activation (59). Experimental evidence highlights FAK as an important player on multiple levels during the metastatic cascade, including the regulation of tumor cell motility and invasion as well 
as tumor angiogenesis (60). Hence, as earlier work suggested, targeting FAK with a small-molecule inhibitor might be beneficial for overcoming adaptive mechanisms following antiangiogenic therapy. Indeed, our in vivo experiments suggested that inhibiting FAK significantly reduced infiltration of platelets and prevented subsequent tumor growth. This has not been shown previously to our knowledge, and, although the small-molecule inhibition used in this study affects both mouse and human cells $(34,35)$, inhibition of platelet infiltration represents an additional mode of action that leads to a reduction in tumor growth.

Overall, our findings have important clinical implications in patients treated with antiangiogenic therapies and provide a mechanism for accelerated tumor growth after cessation of therapy. Our data demonstrate a role for platelet accumulation in the tumor microenvironment following treatment with and withdrawal of antiangiogenic agents and identify FAK inhibitors as a potential regimen to prevent tumor rebound after cessation of antiangiogenic therapy.

\section{Methods}

Reagents. Pazopanib and the FAK inhibitor GSK2256098 were obtained from GlaxoSmithKline under material transfer agreement. Bevacizumab (Avastin) was obtained from Roche and B2O was obtained from Genentech.

Cell lines and siRNA transfections. All cell lines used in this study were obtained from the institutional Cell Line Core laboratory, and, per institutional policy (MD Anderson Cancer Center policy ACA 1044), cell line authentication was performed every year. Additionally, cell lines were routinely tested for mycoplasma contamination using the MycoAlert Kit (Lonza). Human ovarian cancer cell lines HeyA8, SKOV3ip1, OVCAR5, and A2780 were cultured in RPMI-1640 (Sigma-Aldrich) supplemented with $10 \%$ to $15 \%$ FBS and $0.1 \%$ gentamicin sulfate (GeminiBioproducts); murine ovarian cancer cell lines ID8 and ID8-VEGF were grown in DMEM medium supplemented with 5\% FBS and 1\% Insulin-Transferrin-Sodium Selenite (Roche); and the human ovarian cancer cell line 2774 was maintained in MEM medium supplemented with $5 \%$ FBS, $1 \times$ sodium pyruvate $(100 \mathrm{mM}$ stock, Invitrogen), and $1 \times$ nonessential amino acid (Gibco). Cells were maintained at $37^{\circ} \mathrm{C}$ in a humidified incubator infused with $20 \%$ $\mathrm{O}_{2}$ and $5 \% \mathrm{CO}_{2}$. For hypoxia experiments, cells were incubated in an oxygen-controlled hypoxia chamber at $1 \% \mathrm{O}_{2}$. All siRNA transfections were done using RNAiMAX reagent (Invitrogen) using forward transfection protocol, and media were changed after 6 hours.

Mice. Female athymic nude mice were purchased from Taconic Farms, and platelet-specific FAK-deficient mice ( $P f 4-\mathrm{Cr}^{+} \mathrm{Fak}^{f / f l}$ mice) were a gift from Ian Hitchcock (Stony Brook Medicine, New York, New York, USA) (33). Mice were cared for according to guidelines set forth by the American Association for Accreditation of Laboratory Animal Care and the US Public Health Service policy on Human Care and Use of Laboratory Animals. All animals used were 8 to 12 weeks old at the time of cell injection.

Mouse platelet isolation. Platelets were prepared in a manner that would remove plasma contents and nucleated cells. Whole blood was drawn from the inferior vena cava of anesthetized nude mice into a syringe preloaded with 1:9 (vol/vol) 3.8\% sodium citrate and mixed 1:1 (vol/vol) with Tyrodes buffer lacking $\mathrm{Mg}^{2+}$ and $\mathrm{Ca}^{2+}$. Blood was centrifuged at 1,100 rpm $(200 \mathrm{~g})$ for 6 minutes at room temperature. The platelet-rich plasma fraction was passed through a filtration column of Sepharose 2B beads (Sigma-Aldrich) loaded into a siliconized glass column with a 10- $\mu \mathrm{m}$ nylon net filter (Millipore). Sepharose $2 \mathrm{~B}$ beads were previously washed in acetone 1:1 (vol/vol), followed by PBS 1:1 (vol/vol) twice. Cloudy eluents, which contained platelets, were collected. Platelets were counted with a hemocytometer by phase-contrast microscopy at $\times 400$ magnification and immediately used for subsequent experiments. For protein expression analysis, platelets were washed once with PBS and lysed in RIPA buffer containing phosphatase and protease inhibitors.

In vivo experiments. In all the experiments, orthotopic models of ovarian cancer were used as described previously (61). Nude mice were injected with the indicated numbers of human ovarian cancer cells, whereas immunocompetent C57BL/6J WT and Pf4-Cre $\mathrm{Fak}^{\mathrm{fl} / \mathrm{ll}}$ mice were injected with the murine ovarian cancer cell line ID8VEGF. For all i.p. cell injections, cells were harvested at $70 \%$ to $80 \%$ confluence using trypsin-EDTA, neutralized with FBS-containing media, washed, and resuspended to the appropriate cell number in Hanks' balanced salt solution (Gibco) before injection. For SKOV3ip1, A2780, and ID8-VEGF i.p. tumors, $1 \times 10^{6}$ cells in a total volume of $200 \mu \mathrm{l}$ were injected; for HeyA8 tumors, $250 \times 10^{3}$ cells in a total volume of $200 \mu \mathrm{l}$ were injected; and for 2774 tumors, $2 \times 10^{6}$ cells in a total volume of $200 \mu \mathrm{l}$ were injected. For all therapeutic experiments, 10 mice per group were used. Unless indicated otherwise, treatments with pazopanib (100 mg/kg p.o. daily, GlaxoSmithKline) or bevacizumab (6.25 mg/kg i.p. twice weekly, Genentech) were started 1 week after cell injection and either withdrawn after 1 week or continued for approximately 4 weeks until necropsy. 0.5\% hydroxypropylmethyl cellulose/0.1\% Tween-80 and PBS were used as a vehicle control for all pazopanib and bevacizumab/B20 experiments, respectively. In selected experiments, mice were pretreated with pazopanib daily for 7 days prior to cell inoculation to exclude that any preconditioning of the tumor microenvironment by the drug prior to cell injection was responsible for tumor rebound after withdrawal. Platelet transfusions were done twice weekly, and the respective animals received $6 \times 10^{8}$ platelets i.v. For YFP-labeled platelets, platelets were isolated from transgenic C57BL/6 mice expressing YFP under the control of the Pf4 platelet-specific promoter, and $300 \times 10^{6}$ platelets were transfused into tumor-bearing mice. Mice were anesthetized and perfused with $4 \%$ paraformaldehyde. Microscopy for YFP-labeled platelets was performed using a bandpass emission filter for a peak wavelength of $519 \mathrm{~nm}$, given that the emission peak of YFP is $527 \mathrm{~nm}$. To deplete platelets in mice, indicated animals received a rat anti-mouse monoclonal antibody directed against mouse GPIba (R300; Emfret Analytics) twice weekly that caused irreversible Fc-independent platelet depletion within 60 minutes of administration, without inducing platelet activation. A polyclonal nonimmune rat IgG antibody was used as the vehicle control (C301; Emfret Analytics). FAK inhibitor (GSK2256098) was administered daily ( $75 \mathrm{mg} / \mathrm{kg}$, p.o.) in $10 \%$ 1-methyl-2-pyrrolidinone in polyethylene glycol 300 as a carrier for the indicated experiments. The carrier alone was used as a vehicle control. In selected experiments GSK2256098 was given after withdrawal bevacizumab or pazopanib. For all experiments, once mice in any group became moribund they were sacrificed, and their tumors were harvested. Tumor weight and the number of tumor nodules were recorded. Tumor tissue was fixed in formalin for paraffin embedding, frozen in optimal cutting temperature media to prepare frozen slides, 
or snap frozen for lysate preparation. Additionally, blood was collected from the inferior vena cava for subsequent $\mathrm{CBC}$ analysis.

Intravital fixation. At the time of animal sacrifice, mice were anesthetized with isoflurane, an incision was made in the chest cavity allowing visualization of the left heart, and a 21-gauge needle was used to slowly deliver PBS and $4 \%$ paraformaldehyde. In indicated experiments, fixation was preceded by i.v. injection of Dextran (Sigma-Aldrich) or Hypoxyprobe (Hypoxyprobe Inc.) in two mice of each group.

Isolation of immune cells from the spleen. Spleens were harvested from WT or Pf4-Cre $\mathrm{Fak}^{\mathrm{fl} / \mathrm{fl}}$ mice, and splenocytes were released by crushing the spleen through a $40-\mu \mathrm{m}$ filter using the black rubber end of a syringe. Cells were washed once with PBS, and cell suspension was blocked with Mouse BD Fc Block (catalog 553141, BD Biosciences) for 20 minutes on ice. After a washing step using PBS/1\% BSA, cells were incubated with anti-mouse F4/80-PE (catalog 12-4801-80, ebioscience), anti-mouse CD3e-PerCP-Cy5.5 (catalog 45-0031-82, ebioscience), and anti-mouse CD19-PE-Cy7 (catalog 25-0193-81, ebioscience) and diluted 1:200 in PBS/1\%BSA for 30 minutes at $4^{\circ} \mathrm{C}$ in the dark. After washing twice, cells were resuspended in PBS/1\% BSA and transferred to FACS tubes. Cell sorting and collection was performed on a BD Influx cell sorter.

Chitosan nanoparticle preparation. Chitosan nanoparticles were designed as previously described (62). Before in vivo administration, preparation was hydrated with distilled water at room temperature at a concentration of $150 \mu \mathrm{g}$ siRNA per kg per injection. $100 \mu \mathrm{l}$ of siRNA solution was injected i.v. twice weekly.

Immunoblot analysis. Lysates from cultured cells were prepared using modified RIPA buffer (50 mM Tris- $\mathrm{HCl}$ [pH 7.4], $150 \mathrm{mM} \mathrm{NaCl}$, $1 \%$ Triton, $0.5 \%$ deoxycholate) plus phosphatase and proteins inhibitors. The protein concentrations were determined using a BCA Protein Assay Reagent Kit (Pierce Biotechnology). Lysates were loaded and separated on SDS-PAGE. Proteins were transferred to a nitrocellulose membrane by electrophoresis (Bio-Rad Laboratories). Membranes were blocked with either 5\% BSA (for phosphorylated proteins) or $5 \%$ anhydrous milk powder in Tris-buffered saline (for total proteins) for 1 hour and then incubated at $4^{\circ} \mathrm{C}$ overnight with primary antibody (pFAK 1:1,000 [catalog 13009, Cell Signaling], FAK 1:1,000 [catalog 8556, Cell Signaling]). After washing with Tris-buffered saline with Tween 20, the membranes were incubated with HRP-conjugated secondary antibody for 1 hour at room temperature. $\beta$-Actin (ACTB, 1:5,000, catalog A5316, Sigma-Aldrich) was used as a loading control. For evaluation of angiogenesis-related proteins in platelets, we used Mouse Angiogenesis Antibody Array (catalog ARY015, R\&D Systems Inc.) according to the manufacturer's instructions. $150 \mu \mathrm{g}$ platelet protein lysate was used for each group.

qRT-PCR analysis. For mRNA quantification, total RNA was isolated using the Direct-zol RNA MiniPrep Kit (Zymo Research Corp.) and cDNAs were synthesized using the Verso cDNA Kit (Thermo Scientific) per the manufacturers' instructions. Analysis of mRNA levels was performed on a Mx3005P qPCR system (Agilent Technologies). The primers used are as follows: $18 \mathrm{~S}$ fwd: 5'-CGCCGCTAGAGGTGAAATTC-3', 18S rev: 5'-TTGGCAAATGCTTTCGCTC-3', FAK fwd: 5'-CAATGCCTCCAAATTGTCCT-3', and FAK rev: 5'-TCCATCCTCATCCGTTCTTC-3'.

In vitro proliferation and apoptosis assays. Fifty thousand cells were plated in 6-well plates. Media were changed to serum-free media for approximately 24 hours prior to the start of treatment. Platelets were isolated (as described above), and $20 \times 10^{6}$ platelets were added to the cells. After 48 hours (for proliferation) and 72 hours (for apoptosis), the number of proliferating cells was analyzed using the ClickiT EdU Flow Cytometry Kit (Invitrogen) according to the manufacturer's instructions. Apoptosis and cell viability were assessed using Annexin V and 7-amino-actinomycin-D staining (BD Pharmingen), respectively. For indirect coculture of HeyA8 cells and platelets separated by an endothelial monolayer, $1 \times 10^{4} \mathrm{HeyA} 8$ cells were seeded in 24-well plates. At the same time, $1 \times 10^{5}$ human immortalized blood endothelial cells (a gift of Dontscho Kerjaschki, Medical University Vienna, Vienna, Austria) were seeded on a Transwell insert (catalog PIHT12R48, Millipore) after being coating with $20 \mu \mathrm{g} / \mathrm{ml}$ fibronectin (catalog F1141, Sigma-Aldrich). Complete confluency of the endothelial monolayer was checked before pursuing the experiment. The next day HeyA 8 cells were starved overnight, and $10 \times 10^{6}$ platelets were added. The proliferation assay was performed as described above. Experiments were performed 3 times in duplicates.

In vitro invasion. To assess the effect of platelets on cancer cell invasion, a modified Boyden chamber system was used. The wells were separated by a $0.1 \%$ gelatin-coated, cell-permeable membrane. Platelets were isolated and added to a final concentration of $1 \times 10^{8}$ to the lower chamber. Cancer cells $\left(10^{5}\right.$ cells per well $)$ were added to the upper chamber and incubated overnight at $37^{\circ} \mathrm{C}$. After incubation, the cells in the upper chamber were removed with cotton swabs. Cells on membrane were fixed, stained, and counted using light microscopy. Cells from 5 random fields were counted. Experiments were done in duplicate and performed 3 times.

Assessing surface marker expression in platelets after tumor cell coincubation. Platelets were isolated as described above, and approximately $200 \times 10^{6}$ platelets were added to $3 \times 10^{5} \mathrm{HeyA} 8$ cells in a low-attachment plate (catalog 3471, Corning) for 2 hours. Thereafter, cell-platelet mixture was collected, washed once with PBS, and resuspended in $50 \mu$ l of Tyrodes buffer. $5 \mu$ of anti-GPIb $\beta$ Dylight 488 (catalog X488, Emfret) and $5 \mu \mathrm{l}$ of anti-CD62P-PE (catalog M130-2, clone Wug.E9, Emfret) or anti-CD41/CD61-PE (active form, catalog M023-2, clone JON/A, Emfret) was added and incubated for 15 minutes in the dark. Thereafter, $400 \mu$ of PBS was added to stop the staining reaction. As a positive control, platelets were incubated with $0.5 \mathrm{U} / \mathrm{ml}$ of human thrombin (catalog T6884, Sigma-Aldrich) and immunostained accordingly. FACS analysis was done on a Beckman Coulter Gallios Flow Cytometer using forward scatter gating (for particle size) and fluorescence gating $\left(\mathrm{GP} 1 \mathrm{~b} \beta^{+}\right.$particles) to specifically analyze platelets.

Immunohistochemistry and coimmunofluorescence. 5 - $\mu \mathrm{m}$ frozen sections were fixed in acetone, endogenous peroxides were blocked with $3 \%$ hydrogen peroxide in methanol, and nonspecific proteins were blocked with $4 \%$ fish gelatin in Tris-buffered saline for 20 minutes. Proliferation and apoptosis were assessed using anti-Ki67 antibody (catalog RB-90-43-P; Thermo Scientific) and anti-caspase-3 antibody (catalog CP229B; BioCare Medical). For double immunofluorescence, Dylight 488-labeled antibody against the GP1b $\beta$ subunit of the murine platelet/megakaryocyte-specific GPIb-V-IX complex (catalog X488, Emfret) was used in combination with a directly labeled antibody for CD31 (catalog 553373, BD Pharmingen). Hoechst 33342 was used for nuclear counterstain. Slides were mounted using propyl gallate (Fisher Scientific) and glass cover slips. Further, rabbit polyclonal antibody 
against Desmin (catalog ab8592, Abcam) was used to stain pericytes, and rat monoclonal antibody against CD31 (catalog 53370, BD Pharmingen) was used to visualize blood vessels. The number of vessels and pericyte-covered $\mathrm{CD} 31^{+}$vessels and Ki67 and caspase-3 positivity were quantified in 5 randomly selected fields at $\times 200$ magnification. The number of extravasated platelets was determined by counting them in 10 randomly selected fields at $\times 200$ or $\times 400$ magnification.

Transmission electron microscopy. Tumor samples were immersion fixed in $0.1 \mathrm{M}$ sodium cacodylate buffer ( $\mathrm{pH} 7.2$ ) containing $2.5 \%$ glutaraldehyde for 2 hours at room temperature. The tissues were subsequently rinsed in buffer and then post-fixed in $0.1 \mathrm{M}$ sodium cacodylate buffer containing $1 \%$ osmium tetroxide for 1 hour in the dark. The samples were rinsed in distilled water and then dehydrated through an acetone series (30\%, 50\%, 70\%, 90\%, and 100\% acetone) prior to embedding in Embed-812 epoxy resin (Electron Microscopy Sciences). Ultrathin sections (100 nm) were post-stained with uranyl acetate and lead citrate prior to imaging on a Tecnai G2 Spirit BioTWIN (FEI Company) transmission electron microscope.

$A D P$ quantification. The relative levels of ADP in cell culture supernatants or tumor tissue lysates were evaluated using the ADP Colorimetric Assay Kit (Abcam) according to the manufacturer's protocol. Supernatants of normoxic or hypoxic SKOV3ip1 cells from 3 different experiments were used. To quantify ADP levels in tumors, two in vivo samples of each group were used.

Statistics. Statistical analysis was performed using Excel and GraphPad Prism 6. Differences between groups were evaluated using 2-tailed Student's $t$ test or 1-way ANOVA, adjusting for multiple comparisons. Results are presented as the mean \pm SEM. For all statistical analyses, $P<0.05$ was considered statistically significant.

Study approval. All approvals for experiments depicted here were obtained from The University of Texas MD Anderson Cancer Center Institutional Review Board. All animal experiments were approved and supervised by the MD Anderson Cancer Center Institutional Animal Care and Use Committee.

\section{Author contributions}

$\mathrm{MH}$ and JBM performed experiments, analyzed data, and wrote the manuscript. SP, MLT, HJC, JMH, HJD, RLS, MSC, AMN, TG, ASN, KMG, RR, HDH, BZ, GNAP, SYW, and CVP participated in in vitro and in vivo experiments. ARB helped with electron microscopy imaging of tumors. LSM and GLB designed and prepared chi- tosan nanoparticles for in vivo studies. VAK and AKS supervised and designed the study and wrote the manuscript.

\section{Acknowledgments}

Portions of this work were supported by the NIH (CA177909, CA016672, CA109298, UH2TR000943, P50 CA083639, and P50 CA098258), the Cancer Prevention and Research Institute of Texas (RP110595 and RP120214), the Ovarian Cancer Research Fund Inc. (Program Project Development Grant), the RGK Foundation, the Gilder Foundation, the Judi A. Rees Ovarian Cancer Research Fund, Mr. and Mrs. Daniel P. Gordon, the Blanton-Davis Ovarian Cancer Research Program, and the Betty Anne Asche Murray Distinguished Professorship (to A.K. Sood and V. Afshar-Kharghan). M. Haemmerle is supported by a fellowship from the Deutsche Forschungsgemeinschaft. J. Bottsford-Miller, H.J. Dalton, B. Zand, R.L. Stone, and J.M. Hansen were supported by the National Cancer Institute-Department of Health and Human Services-NIH T32 training grant (T32 CA101642). T. Gutschner is supported by the Odyssey Fellowship Program at The University of Texas MD Anderson Cancer Center. S.Y. Wu is supported by the Ovarian Cancer Research Fund Inc., the Foundation for Women's Cancer, and Cancer Prevention Research Institute of Texas training grants (RP101502 and RP101489). R. Rupaimoole was supported in part by the Russell and Diana Hawkins Family Foundation Discovery Fellowship. K.M. Gharpure is supported by the Altman-Goldstein Discovery Fellowship. We thank all members of the MD Anderson Flow Cytometry and Cellular Imaging Core Facility for excellent technical assistance. The core facility is funded by the National Cancer Institute Cancer Center support grant P30CA16672.

Address correspondence to: Anil K. Sood, Department of Gynecologic Oncology and Reproductive Medicine, The University of Texas MD Anderson Cancer Center, 1515 Holcombe Blvd., Unit 1362, Houston, Texas 77030, USA. Phone: 713.745.5266; E-mail: asood@mdanderson.org. Or to: Vahid Afshar-Kharghan, Section of Benign Hematology, The University of Texas MD Anderson Cancer Center, 1515 Holcombe Blvd., Unit 1100, Houston, Texas 77030, USA. Phone: 713.563.7100; E-mail: vakharghan@mdanderson.org.

Hee Dong Han's present address is: Department of Immunology, School of Medicine, Konkuk University, Chungju, South Korea.
1. Folkman J. Tumor angiogenesis: therapeutic implications. N Engl J Med.1971;285(21):1182-1186.

2. Blagosklonny MV. Antiangiogenic therapy and tumor progression. Cancer Cell. 2004;5(1):13-17.

3. Choi HJ, Armaiz Pena GN, Pradeep S, Cho MS, Coleman RL, Sood AK. Anti-vascular therapies in ovarian cancer: moving beyond anti-VEGF approaches. Cancer Metastasis Rev. 2015;34(1):19-40.

4. Hurwitz H, et al. Bevacizumab plus irinotecan, fluorouracil, and leucovorin for metastatic colorectal cancer. N Engl J Med. 2004;350(23):2335-2342.

5. Miller K, et al. Paclitaxel plus bevacizumab versus paclitaxel alone for metastatic breast cancer. N Engl J Med. 2007;357(26):2666-2676.

6. Loges S, Mazzone M, Hohensinner P, Carmeliet P. Silencing or fueling metastasis with VEGF inhibitors: antiangiogenesis revisited. Cancer Cell. 2009;15(3):167-170.

7. Welti J, Loges S, Dimmeler S, Carmeliet P. Recent molecular discoveries in angiogenesis and antiangiogenic therapies in cancer. J Clin Invest. 2013;123(8):3190-3200.

8. Perren TJ, et al. A phase 3 trial of bevacizumab in ovarian cancer. $N$ Engl J Med. 2011;365(26):2484-2496.

9. Oza AM, et al. Standard chemotherapy with or without bevacizumab for women with newly diagnosed ovarian cancer (ICON7): overall survival results of a phase 3 randomised trial. Lancet Oncol. 2015;16(8):928-936.

10. Burger RA, et al. Incorporation of bevacizumab in the primary treatment of ovarian cancer. $\mathrm{NEnglJ}$ Med. 2011;365(26):2473-2483.
11. Griffioen AW, et al. Rapid angiogenesis onset after discontinuation of sunitinib treatment of renal cell carcinoma patients. Clin Cancer Res. 2012;18(14):3961-3971.

12. Mancuso MR, et al. Rapid vascular regrowth in tumors after reversal of VEGF inhibition. J Clin Invest. 2006;116(10):2610-2621.

13. Sharpe K, et al. The effect of VEGF-targeted therapy on biomarker expression in sequential tissue from patients with metastatic clear cell renal cancer. Clin Cancer Res. 2013;19(24):6924-6934.

14. Ebos JM, Lee CR, Cruz-Munoz W, Bjarnason GA, Christensen JG, Kerbel RS. Accelerated metastasis after short-term treatment with a potent inhibitor of tumor angiogenesis. Cancer Cell. 2009;15(3):232-239.

15. Rubenstein JL, et al. Anti-VEGF antibody treat- 
ment of glioblastoma prolongs survival but results in increased vascular cooption. Neoplasia. 2000;2(4):306-314.

16. Paez-Ribes M, et al. Antiangiogenic therapy elicits malignant progression of tumors to increased local invasion and distant metastasis. Cancer Cell. 2009;15(3):220-231.

17. Riess L. Zur pathologischen Anatomie des Blutes. Arch Anat Physiol Wissensch Med. 1872;39:237-249.

18. Trousseau ABV, Cormack J. Lectures On Clinical Medicine, Delivered At The Hôtel-Dieu, Paris. London, United Kingdom: R Hardwicke; 1867.

19. Cho MS, et al. Platelets increase the proliferation of ovarian cancer cells. Blood. 2012;120(24):4869-4872.

20. Bottsford-Miller J, et al. Differential platelet levels affect response to taxane-based therapy in ovarian cancer. Clin Cancer Res. 2015;21(3):602-610.

21. Labelle M, Begum S, Hynes RO. Direct signaling between platelets and cancer cells induces an epithelial-mesenchymal-like transition and promotes metastasis. Cancer Cell. 2011;20(5):576-590.

22. Labelle M, Begum S, Hynes RO. Platelets guide the formation of early metastatic niches. Proc Natl Acad Sci U S A . 2014;111(30):E3053-E3061.

23. Stone RL, et al. Paraneoplastic thrombocytosis in ovarian cancer. N Engl JMed. 2012;366(7):610-618.

24. Klement GL, et al. Platelets actively sequester angiogenesis regulators. Blood. 2009;113(12):2835-2842.

25. Pinedo HM, Verheul HM, D’Amato RJ, Folkman J. Involvement of platelets in tumour angiogenesis? Lancet. 1998;352(9142):1775-1777.

26. Stone R, et al. Unraveling the century-old mystery of paraneoplastic thrombocytosis in ovarian cancer. Gynecol Oncol. 2012;127(1 suppl):S2.

27. Noto T, et al. Temporal and topographic profiles of tissue hypoxia following transient focal cerebral ischemia in rats. J Vet Med Sci. 2006;68(8):803-807.

28. Serebrovskaya TV, Nikolsky IS, Nikolska VV, Mallet RT, Ishchuk VA. Intermittent hypoxia mobilizes hematopoietic progenitors and augments cellular and humoral elements of innate immunity in adult men. High Alt Med Biol. 2011;12(3):243-252.

29. Oga T, et al. Effects of obstructive sleep apnea with intermittent hypoxia on platelet aggregability. J Atheroscler Thromb. 2009;16(6):862-869.

30. Raju NC, Eikelboom JW, Hirsh J. Platelet ADPreceptor antagonists for cardiovascular disease: past, present and future. Nat Clin Pract Cardiovasc Med. 2008;5(12):766-780.

31. Bastida E, Ordinas A, Giardina SL, Jamieson GA. Differentiation of platelet-aggregating effects of human tumor cell lines based on inhibition studies with apyrase, hirudin, and phospholipase. Cancer Res. 1982;42(11):4348-4352.

32. Egan K, et al. Platelet adhesion and degranulation induce pro-survival and pro-angiogenic signalling in ovarian cancer cells. PLoS One. 2011;6(10):e26125.

33. Hitchcock IS, Fox NE, Prevost N, Sear K, Shattil SJ, Kaushansky K. Roles of focal adhesion kinase (FAK) in megakaryopoiesis and platelet function: studies using a megakaryocyte lineage specific FAK knockout. Blood. 2008;111(2):596-604.

34. Thanapprapa D, et al. PTEN expression as a predictor of response to focal adhesion kinase inhibition in uterine cancer. Mol Cancer Ther. 2015;14(6):1466-1475.

35. Stone RL, et al. Focal adhesion kinase: an alternative focus for anti-angiogenesis therapy in ovarian cancer. Cancer Biol Ther. 2014;15(7):919-929.

36. Schultze A, Fiedler W. Clinical importance and potential use of small molecule inhibitors of focal adhesion kinase. Anticancer Agents Med Chem. 2011;11(7):593-599.

37. Zhang J, He DH, Zajac-Kaye M, Hochwald SN. A small molecule FAK kinase inhibitor, GSK2256098, inhibits growth and survival of pancreatic ductal adenocarcinoma cells. Cell Cycle. 2014;13(19):3143-3149.

38. Pujade-Lauraine E, et al. Bevacizumab combined with chemotherapy for platinum-resistant recurrent ovarian cancer: The AURELIA openlabel randomized phase III trial. J Clin Oncol. 2014;32(13):1302-1308.

39. Stockler MR, et al. Patient-reported outcome results from the open-label phase III AURELIA trial evaluating bevacizumab-containing therapy for platinum-resistant ovarian cancer. J Clin Oncol. 2014;32(13):1309-1316.

40. Tomao F, Tomao S, Benedetti Panici P. Combination of bevacizumab and chemotherapy for platinum-resistant recurrent ovarian cancer: some observations about the AURELIA trial JClin Oncol. 2014;32(31):3580.

41. Katsumata N, et al. Dose-dense paclitaxel once a week in combination with carboplatin every 3 weeks for advanced ovarian cancer: a phase 3 , open-label, randomised controlled trial. Lancet. 2009;374(9698):1331-1338.

42. McGuire WP, et al. Cyclophosphamide and cisplatin compared with paclitaxel and cisplatin in patients with stage III and stage IV ovarian cancer. NEngl JMed. 1996;334(1):1-6.

43. Bennouna J, et al. Continuation of bevacizumab after first progression in metastatic colorectal cancer (ML18147): a randomised phase 3 trial. Lancet Oncol. 2013;14(1):29-37.

44. von Minckwitz G, et al. Bevacizumab plus chemotherapy versus chemotherapy alone as second-line treatment for patients with HER2-negative locally recurrent or metastatic breast cancer after firstline treatment with bevacizumab plus chemotherapy (TANIA): an open-label, randomised phase 3 trial. Lancet Oncol. 2014;15(11):1269-1278.

45. De Bock K, Mazzone M, Carmeliet P. Antiangiogenic therapy, hypoxia, and metastasis: risky liaisons, or not? Nat Rev Clin Oncol. 2011;8(7):393-404.
46. Bagri A, et al. Effects of anti-VEGF treatment duration on tumor growth, tumor regrowth, and treatment efficacy. Clin Cancer Res. 2010;16(15):3887-3900.

47. Fidler IJ. Angiogenic heterogeneity: regulation of neoplastic angiogenesis by the organ microenvironment. J Natl Cancer Inst. 2001;93(14):1040-1041.

48. Jain RK. Normalization of tumor vasculature: an emerging concept in antiangiogenic therapy. Science. 2005;307(5706):58-62.

49. Nagy JA, Chang SH, Shih SC, Dvorak AM, Dvorak HF. Heterogeneity of the tumor vasculature. Semin Thromb Hemost. 2010;36(3):321-331.

50. Casanovas O, Hicklin DJ, Bergers G, Hanahan D. Drug resistance by evasion of antiangiogenic targeting of VEGF signaling in late-stage pancreatic islet tumors. Cancer Cell. 2005;8(4):299-309.

51. Fernando NT, et al. Tumor escape from endogenous, extracellular matrix-associated angiogenesis inhibitors by up-regulation of multiple proangiogenic factors. Clin Cancer Res. 2008;14(5):1529-1539.

52. Franco M, et al. Targeted anti-vascular endothelial growth factor receptor-2 therapy leads to short-term and long-term impairment of vascular function and increase in tumor hypoxia. Cancer Res. 2006;66(7):3639-3648.

53. Levin J, Conley CL. Thrombocytosis associated with malignant disease. Arch Intern Med. 1964;114:497-500.

54. Borsig L. The role of platelet activation in tumor metastasis. Expert Rev Anticancer Ther. 2008;8(8):1247-1255

55. Noto T, et al. Tacrolimus (FK506) limits accumulation of granulocytes and platelets and protects against brain damage after transient focal cerebral ischemia in rat. Biol Pharm Bull. 2007;30(2):313-317.

56. Qi C, et al. P-Selectin-mediated adhesion between platelets and tumor cells promotes intestinal tumorigenesis in $\mathrm{Apc}(\mathrm{Min} /+)$ mice. Int JBiol Sci. 2015;11(6):679-687.

57. Pertuy F, et al. Broader expression of the mouse platelet factor 4-cre transgene beyond the megakaryocyte lineage. J Thromb Haemost. 2015;13(1):115-125.

58. Thijs T, Deckmyn H, Broos K. Model systems of genetically modified platelets. Blood. 2012;119(7):1634-1642.

59. Auger KR, et al. 387 The focal adhesion kinase inhibitor GSK2256098: a potent and selective inhibitor for the treatment of cancer. Eur JCancer. 2012;48:118.

60. Golubovskaya VM. Focal adhesion kinase as a cancer therapy target. Anti-cancer agents in medicinal chemistry. 2010;10(10):735-741.

61. Rupaimoole R, et al. Hypoxia-mediated downregulation of miRNA biogenesis promotes tumour progression. Nat Commun. 2014;5:5202.

62. Lu C, et al. Regulation of tumor angiogenesis by EZH2. Cancer Cell. 2010;18(2):185-197. 